\title{
Corrections
}

\section{Correction: Joiner and Green et al., "Primary Cilia on Horizontal Basal Cells Regulate Regeneration of the Olfactory Epithelium"}

In the article "Primary Cilia on Horizontal Basal Cells Regulate Regeneration of the Olfactory Epithelium" by Ariell M. Joiner, Warren W. Green, Jeremy C. McIntyre, Benjamin L. Allen, James E. Schwob, and Jeffrey R. Martens, which appeared on pages 13761-13772 of the October 7, 2015 issue, the authors regret an error in Figure 3C. The error in Figure $3 C$ had the images for both Control and iHBC-IFT88 erroneously duplicated from Figure 5A. Figure $3 C$ has been corrected to include images of Arl13b-labeled cilia on K5-labeled horizontal basal cells (HBCs) for both Control and iHBC-IFT88 tissues examined after 4 weeks of administration of dox-containing diet (P28 +4 weeks). Figure $5 A$ is unaffected by this error and remains as is. The error in Figure $3 C$ does not affect the corresponding text and figure caption, nor the interpretations and conclusions of our paper. Figure 3 has been corrected in the online PDF version and is displayed below.
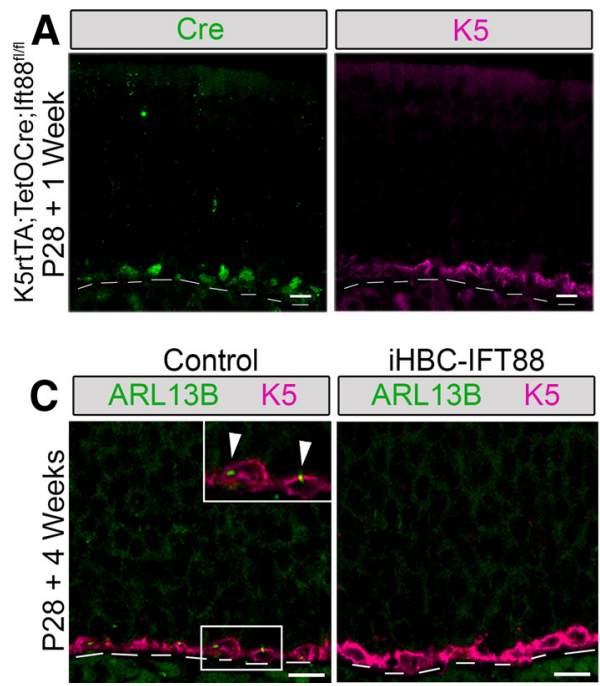
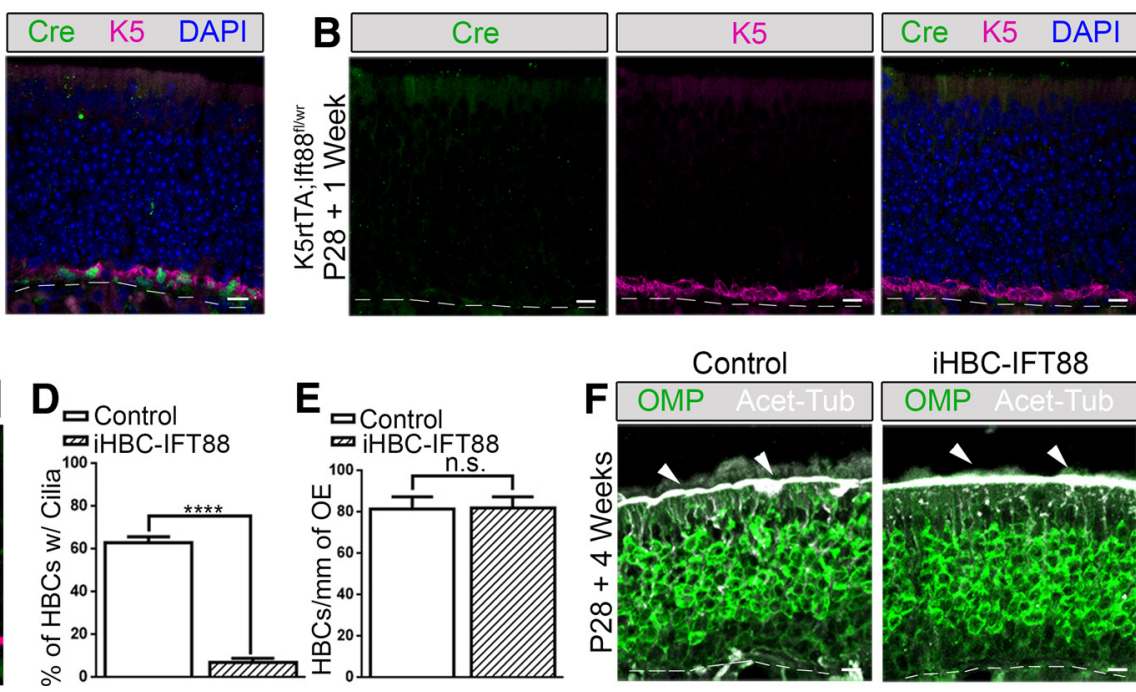

Figure 3.

\section{Correction: Joo et al., "Separate Perceptual and Neural Processing of Velocity- and Disparity-Based 3D Motion Signals"}

In the article "Separate Perceptual and Neural Processing of Velocity- and Disparity-Based 3D Motion Signals" by Sung Jun Joo, Thaddeus B. Czuba, Lawrence K. Cormack, and Alexander C. Huk, which appeared on pages 10791-10802 of the October 16, 2016 issue, the authors regret an error in which time series labels were transposed in the caption of Figure $2 A$. The fourth sentence of the caption should be corrected as follows: "Blue data points represent fMRI responses to the same-direction tests (i.e., test motion in the same direction as adaptation); red data points represent responses to the opposite-direction tests." This brings the caption in line with figure descriptions in the main text, which are correct as published. This error does not affect any of the results, conclusions, or interpretations. The figure legend has been corrected on the online PDF version. 